\title{
Failure of testosterone replacement therapy to improve symptoms correlates with burden of systemic conditions
}

\author{
Nicholas J. Farber", Sarah C. Vij", Daniel A. Shoskes \\ Department of Urology, Glickman Urologic and Kidney Institute, Cleveland Clinic Foundation, Cleveland, OH, USA \\ Contributions: (I) Conception and design: DA Shoskes, SC Vij; (II) Administrative support: All authors; (III) Provision of study materials or patients: \\ DA Shoskes, SC Vij; (IV) Collection and assembly of data: DA Shoskes, SC Vij; (V) Data analysis and interpretation: All authors; (VI) Manuscript \\ writing: All authors; (VII) Final approval of manuscript: All authors. \\ \#These authors contributed equally to this work. \\ Correspondence to: Nicholas J. Farber, MD. Department of Urology, Glickman Urologic and Kidney Institute, Cleveland Clinic Foundation, 9500 \\ Euclid Avenue Q10, Cleveland, OH 44195, USA. Email: farbern@ccf.org.
}

Background: The symptoms of hypogonadism are non-specific and restoring testosterone (T) to physiologic levels may not lead to clinical improvement. In men with a high burden of systemic illness, it is difficult to assess whether hypogonadism is a primary contributing factor of their symptoms. Given that testosterone replacement therapy (TRT) is not without risk, it is important to understand which patients will benefit from treatment. Therefore, we hypothesize that men with a higher burden of systemic illness would be less likely to continue with TRT.

Methods: We performed a retrospective review of our men's health registry for men who started TRT and adhered to follow up labs and visits within the first year. We restricted treatment to Testopel pellets due to reliable early $\mathrm{T}$ levels. Men were classified as yes/no for continued TRT based on whether they felt their presenting symptoms improved on therapy and they chose to continue TRT. The previously validated ACTIONS men's health phenotype was calculated as a composite systemic disease score grading severity [0-2] for each of anxiety, cardiovascular disease, low testosterone, diabetes, obesity, neurologic disease and obstructive sleep apnea (total score 0-14).

Results: Sixty men were identified with a mean age of 59.5 (range, 33-81) years and mean starting total testosterone of 215 [48-332] ng/dL. Thirty-nine men (65\%) felt symptomatic benefit and continued therapy for a median of $40.4(20.5-76.4)$ months vs. 21 men without benefit treated for a median of 4.1 months (2.9-10.7, $\mathrm{P}<0.0001)$. Those who stopped TRT had a higher ACTIONS score than those who continued $(8 \pm 2.5$ vs. $4.1 \pm 1.6, \mathrm{P}<0.0001)$. Age weakly correlated with total ACTIONS score $(\mathrm{r}=0.28, \mathrm{P}=0.03)$ but age had no impact on continuing TRT and the relationship between continuing TRT and ACTIONS score held true regardless of age.

Conclusions: Patients with a greater burden of systemic disease were less likely to have symptomatic improvement with TRT and more likely to stop therapy within a year. As several hypogonadal symptoms are non-specific, it is imperative that patients be counseled on the likelihood of success with TRT, particularly if they have multiple comorbidities. Ideal outcomes may come from multimodal therapy that includes lifestyle modification, and optimization of conditions such as diabetes, cardiovascular disease and sleep apnea.

Keywords: Testosterone; men's health; testosterone pellets; comorbidity

Submitted Dec 09, 2019. Accepted for publication Apr 26, 2020.

doi: $10.21037 /$ tau-19-848

View this article at: http://dx.doi.org/10.21037/tau-19-848 


\section{Introduction}

Hypogonadism is a highly prevalent condition, affecting approximately $40 \%$ of men aged 45 or older (1) and manifesting as a constellation of signs and symptoms including low libido, erectile dysfunction, fatigue, decreased lean body mass, increased body fat, and decreased bone density. Men seeking care for these symptoms often have a high burden of systemic disease, making it challenging to determine whether restoring testosterone $(\mathrm{T})$ to normal serum levels will improve symptoms (1). Complicating the treatment planning is the fact that the symptoms of low testosterone are non-specific and may share symptoms with other comorbid conditions. Accordingly, in patients with medical complexity, it is difficult to predict who will derive symptom benefit from testosterone replacement therapy (TRT). This is distinct from the population of hypogonadal men with comorbidities that could be improved by testosterone normalization without symptoms, such as those with HIV/AIDS, chronic corticosteroid use, or poorly controlled type II diabetes (2).

A recently developed and validated comorbidity phenotype score (ACTIONS) has been shown to correlate systemic health conditions with urologic symptoms (3), including erectile dysfunction, lower urinary tract symptoms, and pelvic pain. The ACTIONS score is a composite score of seven subsections (anxiety, cardiovascular disease, testosterone, insulin/diabetes, obesity, neurologic disorders, and sleep apnea), with each domain independently associated with urologic symptoms.

To date, there is no literature analyzing the impact of comorbidity burden on outcomes of TRT. We hypothesize that hypogonadal men with a higher burden of systemic disease as measured by a higher ACTIONS score are more likely to discontinue TRT due to lack of symptom improvement. We primarily sought to correlate total ACTIONS score with response to TRT, with a secondary aim of identifying associations between ACTIONS subdomains and response to TRT.

\section{Methods}

After institutional review board approval, a retrospective review of the electronic medical record was performed to identify all patients who underwent TRT with TESTOPEL $^{\circledR}$ (testosterone pellets) administered by a single urologist from 2011 to 2015 . We restricted treatment to testosterone pellets due to reliable sustained $\mathrm{T}$ normalization without much need for dose adjustment.
Patients were excluded from the study for the following: non-adherence to monitoring labs, no follow-up visits for the first year after starting TRT, or missing documentation of comorbidities and systemic conditions. Men were administered testosterone pellets in the office setting, with repeat pellet administration about every 4 months. All men were started on 12 Testopel pellets with laboratory values measured at 6 and 12 weeks initially.

After starting TRT, men were classified as "continued TRT" based on subjective improvement of symptoms on therapy and continuing TRT versus "discontinued TRT" based on lack of symptom improvement and a discontinuation of TRT. All patients were assigned an ACTIONS men's health phenotype score (total score 0-14), calculated as the sum of the systemic disease score from each of the following subdomains $(0=$ mild, $1=$ moderate, and $2=$ severe): anxiety/depression, cardiovascular disease, low testosterone, insulin/diabetes, obesity, neurologic disease and obstructive sleep apnea. Improvement on TRT was defined as continuation of therapy with normalization of serum testosterone levels.

\section{Results}

Sixty men were included in the study. Their mean age was 59.5 (range, 33-81) years and mean starting total testosterone of 215 [48-332] ng/dL. Total ACTIONS score ranged from 2-12 (median, 5). Concomitant symptomatic BPH was present in $36.7 \%$ of the cohort and $76.7 \%$ complained of erectile dysfunction.

Thirty-nine men $(65 \%)$ felt symptomatic improvement and continued therapy for a median follow up of 40.4 (range, 20.5-76.4) months. Twenty-one men (35\%) did not have symptomatic improvement and were treated for a median of 4.1 (range, 2.9-10.7) months. There was no significant difference in mean age (continued: $58.1 v s$. discontinued: 62.5 years; $\mathrm{P}=0.100$ ) or pre-treatment testosterone values (continued: $209.4 v s$. discontinued $225.5 \mathrm{ng} / \mathrm{dL} ; \mathrm{P}=0.296$ ) between the continued and discontinued TRT cohorts. The mean ACTIONS score in the cohort who stopped TRT was $8 \pm 2.5$ as compared to the mean ACTIONS score of $4.1 \pm 1.6$ in the group that continued TRT $(\mathrm{P}<0.0001)$, as demonstrated in Figure 1. Age correlated with systemic score $(\mathrm{r}=0.28, \mathrm{P}=0.03)$ but did not impact response to pellets $(\mathrm{P}=0.14)$ (Figure 2).

\section{Discussion}

Testosterone levels decline with age at a rate of approximately 
$1-2 \%$ per year (4) but many men never achieve a drop low enough to cause symptoms. Indeed, symptoms attributable to low $\mathrm{T}$ such as fatigue, depression, difficulty concentrating, loss of muscle mass and erectile dysfunction can have multiple other etiologies. Nevertheless, TRT has grown considerably in recent years, with a more than 3 -fold increase in testosterone prescriptions from 2001 to 2011 (5). In men with hypogonadism, there is often a higher prevalence of systemic comorbidities, including diabetes mellitus (6), obesity (7), and cardiovascular disease (8). Given that the symptoms in hypogonadal men are non-specific in nature, men with additional comorbidities may present with symptoms that are due to hypogonadism or their comorbid conditions or

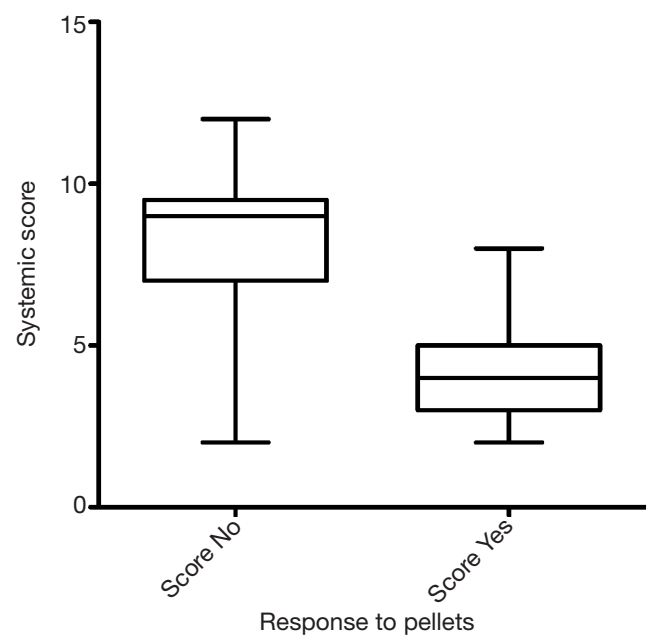

Figure 1 Plot showing response to testosterone pellets as compared to systemic score $(\mathrm{P}<0.0001)$.

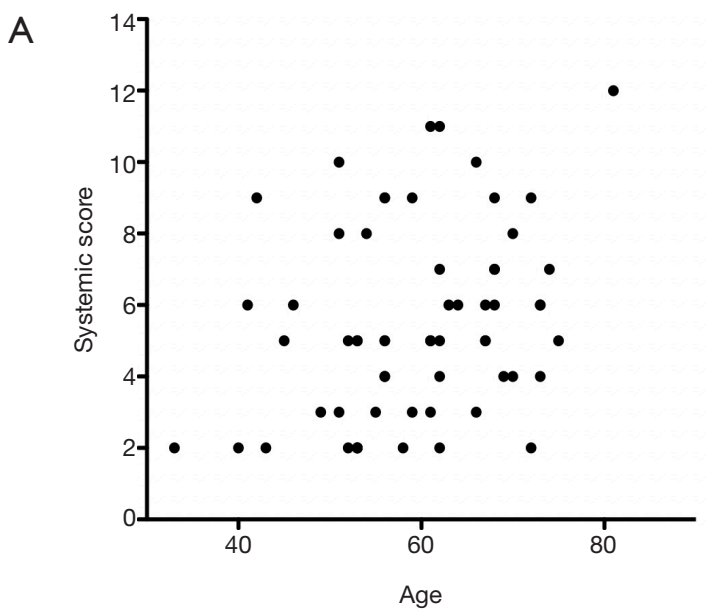

both. Thus, TRT may lead to a non-response in certain hypogonadal men with symptoms primarily driven by their comorbidities rather than hypogonadism. The clinical challenge is in identifying this population.

Recently, a health phenotype score termed ACTIONS was developed, which was validated as an instrument that correlates comorbidity burden with urologic symptoms. One recent application of this phenotype was a study showing that a lower ACTIONS score correlated with medication discontinuation after transurethral resection of the prostate (TURP) (9). This suggests that patients with lower total burden of systemic comorbidities were more likely to respond to TURP and not require additional medications. Similarly, we sought to understand whether ACTIONS score correlated with TRT continuation or discontinuation. We hypothesized that hypogonadal men with greater comorbidity burden, as measured via ACTIONS score, are more likely to discontinue TRT due to lack of symptom improvement.

In our retrospective study of 60 men undergoing testosterone pellet insertion, 39 men continued TRT due to symptomatic improvement and 21 men were non-responders and discontinued TRT. There were no differences between the groups in clinicodemographic variables or pre-treatment testosterone levels. Interestingly, there was a significant difference in ACTIONS score between the group who stopped TRT compared to the group who continued TRT. This suggests that patients with lower total burden of systemic comorbidities were more likely to respond to and continue TRT. Presumably, those patients with a higher burden of comorbidities experienced symptoms related to

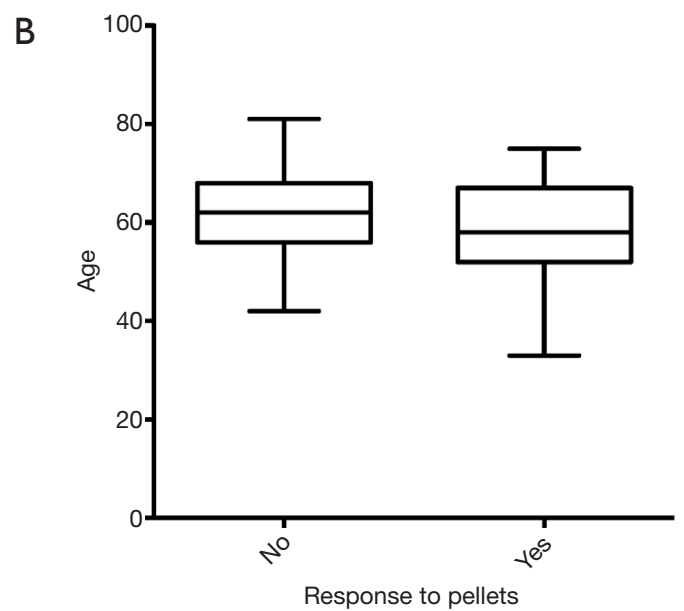

Figure 2 Plot showing: (A) correlation between age and systemic score $(\mathrm{r}=0.28, \mathrm{P}=0.03)$; $(\mathrm{B})$ the response to pellets based on age $(\mathrm{P}=0.14)$. 
their comorbidities rather than their hypogonadal state, explaining the lack of symptomatic improvement upon normalization of testosterone levels. In terms of other factors predicting response to TRT, age correlated with systemic score but did not impact response to pellets.

The potential to identify non-responders to TRT is of significant clinical importance. Although the most robust evidence does not clearly support the notion that TRT increases risk of cardiovascular and thromboembolic events, prostate cancer, or benign prostatic hyperplasia, the FDA mandates a warning for these side effects (10-12). Therefore, the ability to predict patients least likely to derive benefit from TRT helps minimize any potential side effects, especially in the subset with a proven high burden of comorbidities.

Limitations to this study include its retrospective nature and incomplete follow up due to a significant non-regional patient population at a tertiary care center. Another limitation is that the only testosterone formulation we included in our analysis was pellets. However, testosterone pellets were chosen as the formulation of choice for this study due to reliable and sustained serum testosterone levels. Pharmacokinetic data by Kaminetsky et al. shows that testosterone pellets provide therapeutic testosterone levels in $100 \%$ of patients at 4 weeks and even $86 \%$ of patients at 12 weeks (13). We sought to choose a formulation with reliable serum $T$ levels such that the patient decision for discontinuation of testosterone was not due to inadequate therapy, but rather due to a true lack of symptomatic improvement. Conversely, formulations such as injections often have more labile serum $\mathrm{T}$ concentrations and may require more dose adjustment in the initial period; these formulary drawbacks resulting in a failure to normalize testosterone levels may confound an assessment of whether a patient is symptomatically benefitting from TRT. Other limitations of the study include defining improvement as continuation of therapy. Occasionally, patients may desire to continue therapy even without significant symptomatic improvement. However, as a policy, we did not offer additional TRT to patients who did not respond to their initial TRT trial. Overall, our study is novel in that it provides preliminary data suggesting the ACTIONS score may discriminate between patients who respond or do not respond to TRT based on their comorbidities.

\section{Conclusions}

Patients with a higher burden of systemic disease were less likely to have symptomatic improvement and more likely to stop TRT within the first year. Patients should be counseled on likelihood of successful treatment with TRT, particularly that the higher their systemic burden of disease, the less likely they are to have symptomatic improvement with therapy. Multimodal therapy to control systemic comorbidities could lead to improved success with TRT and should be targeted as an area of future investigation.

\section{Acknowledgments}

Funding: None.

\section{Footnote}

Data Sharing Statement: Available at http://dx.doi. org/10.21037/tau-19-848

Conflicts of Interest: All authors have completed the ICMJE uniform disclosure form (available at http://dx.doi. org/10.21037/tau-19-848). DAS reports other from Urogen, other from Farr labs, other from Triurol, outside the submitted work. The other authors have no conflicts of interest to declare.

Ethical Statement: The authors are accountable for all aspects of the work in ensuring that questions related to the accuracy or integrity of any part of the work are appropriately investigated and resolved. The study was approved by the institutional review board: IRB 17-401. Since only a medical record review was performed, this study was exempt from informed consent.

Open Access Statement: This is an Open Access article distributed in accordance with the Creative Commons Attribution-NonCommercial-NoDerivs 4.0 International License (CC BY-NC-ND 4.0), which permits the noncommercial replication and distribution of the article with the strict proviso that no changes or edits are made and the original work is properly cited (including links to both the formal publication through the relevant DOI and the license). See: https://creativecommons.org/licenses/by-nc-nd/4.0/.

\section{References}

1. Mulligan T, Frick MF, Zuraw QC, et al. Prevalence of hypogonadism in males aged at least 45 years: the HIM study. Int J Clin Pract 2006;60:762-9. 
2. Mulhall JP, Trost LW, Brannigan RE, et al. Evaluation and management of testosterone deficiency: AUA guideline. J Urol 2018;200:423-32.

3. Shoskes DA, Vij SC, Shoskes A, et al. Development of a clinically relevant men's health phenotype and correlation of systemic and urologic conditions. Urology 2018;114:77-82.

4. Stanworth RD, Jones TH. Testosterone for the aging male; current evidence and recommended practice. Clin Interv Aging 2008;3:25-44.

5. Baillargeon J, Urban RJ, Ottenbacher KJ, et al. Trends in androgen prescribing in the United States, 2001 to 2011. JAMA Intern Med 2013;173:1465-6.

6. Betancourt-Albrecht M, Cunningham GR. Hypogonadism and diabetes. Int J Impot Res 2003;15 Suppl 4:S14-20.

7. Grossmann M. Hypogonadism and male obesity: focus on unresolved questions. Clin Endocrinol (Oxf) 2018;89:11-21.

8. Zarotsky V, Huang MY, Carman W, et al. Systematic literature review of the risk factors, comorbidities, and consequences of hypogonadism in men. Andrology 2014;2:819-34.

9. Zhang JH, Gill BA, Wilkins L, et al. Systemic comorbidity

Cite this article as: Farber NJ, Vij SC, Shoskes DA. Failure of testosterone replacement therapy to improve symptoms correlates with burden of systemic conditions. Transl Androl Urol 2020;9(3):1108-1112. doi:10.21037/tau-19-848 burden using the actions phenotype predicts urologic medication discontinuation following transurethral resection of the prostate. Urology 2019;127:91-6.

10. Marks LS, Mazer NA, Mostaghel E, et al. Effect of testosterone replacement therapy on prostate tissue in men with late-onset hypogonadism: a randomized controlled trial. JAMA 2006;296:2351-61.

11. Finkle WD, Greenland S, Ridgeway GK, et al. Increased risk of non-fatal myocardial infarction following testosterone therapy prescription in men. PLoS One 2014;9:e85805.

12. FDA Drug Safety Communication: FDA cautions about using testosterone products for low testosterone due to aging; requires labeling change to inform of possible increased risk of heart attack and stroke with use. 2015. Available online: http://www.fda.gov/Drugs/DrugSafety/ ucm436259.htm

13. Kaminetsky JC, Moclair B, Hemani M, et al. A phase IV prospective evaluation of the safety and efficacy of extended release testosterone pellets for the treatment of male hypogonadism. J Sex Med 2011;8:1186-96. 